\title{
Equation of state for hot QCD and compact stars from a mean-field approach
}

\author{
Anton Motornenko $\odot,{ }^{1,2}$ Jan Steinheimer, ${ }^{2}$ Volodymyr Vovchenko, ${ }^{1,2}$ Stefan Schramm, ${ }^{1,2,{ }^{*}}$ and Horst Stoecker ${ }^{1,2,3}$ \\ ${ }^{1}$ Institut für Theoretische Physik, Goethe Universität, D-60438 Frankfurt am Main, Germany \\ ${ }^{2}$ Frankfurt Institute for Advanced Studies, Giersch Science Center, D-60438 Frankfurt am Main, Germany \\ ${ }^{3}$ GSI Helmholtzzentrum für Schwerionenforschung GmbH, D-64291 Darmstadt, Germany
}

(Received 6 June 2019; revised manuscript received 29 November 2019; accepted 19 February 2020; published 9 March 2020)

\begin{abstract}
The thermodynamic properties of high temperature and high density QCD matter are explored within the chiral SU(3)-flavor parity-doublet Polyakov-loop quark-hadron mean-field model, CMF. The quark sector of the CMF model is tuned to describe the $\mu_{B}=0$ thermodynamics data of lattice QCD. The resulting lines of constant physical variables as well as the baryon number susceptibilities are studied in some detail in the temperaturechemical-potential plane. The CMF model predicts three consecutive transitions: the nuclear first-order liquidvapor phase transition, chiral symmetry restoration, and the crossover transition to a quark matter phase. All three phenomena are crossovers, for most of the $T-\mu_{B}$ plane. The deviations from the free ideal hadron gas baseline at $\mu_{B}=0$ and $T \approx 100-200 \mathrm{MeV}$ can be attributed to remnants of the liquid-vapor first-order phase transition in nuclear matter. The chiral crossing transition determines the baryon fluctuations at much higher $\mu_{B} \approx 1.5 \mathrm{GeV}$. At high baryon densities, $\mu_{B} \approx 2.4 \mathrm{GeV}$, the behavior of fluctuations is controlled by crossover to quark matter. The CMF model also describe well the static properties of high $\mu_{B}$ neutron stars as well as recent neutron star merger observations. The effective equation of state presented here describes simultaneously lattice QCD results at $\mu_{B}=0$ as well as observed physical phenomena (nuclear matter and neutron star matter) at $T \cong 0$ and high densities, $\mu_{B}>1 \mathrm{GeV}$.
\end{abstract}

DOI: 10.1103/PhysRevC.101.034904

\section{INTRODUCTION}

At low temperatures and high baryonic densities a transition from hadronic matter to a cold deconfined state with quark degrees of freedom is expected [1]; this transition is supported by perturbative QCD calculations [2]. This phenomenon is a particular case of appearance of a deconfined state as in quark-gluon plasma (QGP) which is expected at large temperatures [3]. The study of the unknown QCD phase diagram is one of the main motivations for the state-of-theart research in the nuclear and particle physics community. A rich phase structure is conjectured for finite temperatures and chemical potentials [4-8]. The QCD phase structure is an important ingredient for the understanding of the early universe, of ultrarelativistic heavy ion collisions, and of the evolution, structure and inspirals of neutron stars [9]. Even though QCD is a well-established theory with only a few parameters, perturbative calculations are inappropriate in the crossover regions of $T$ and $\mu_{B}$ discussed here due to the large

\footnotetext{
${ }^{*}$ Deceased.
}

Published by the American Physical Society under the terms of the Creative Commons Attribution 4.0 International license. Further distribution of this work must maintain attribution to the author(s) and the published article's title, journal citation, and DOI. Funded by $S C O A P^{3}$. values of the QCD coupling constant at scales relevant for most of these applications [10]. The infamous sign problem [11] prohibits lattice QCD (LQCD) calculations at finite densities.

The running experiments at the Large Hadron Collider (LHC), at the Relativistic Heavy Ion Collider (RHIC), at the Super Proton Synchrotron (SPS), and at the Heavy Ion Synchrotron (SIS) provide state-of-the-art data of the measurements of properties of matter produced in heavy-ion collisions (HIC). The detailed information of the particle production as measured in these experiments allows us to extract both thermodynamic and kinetic characteristics of the system that is created.

Astrophysical observations of compact stars, along with data from the recent gravitational-wave detection by LIGO and VIRGO collaborations [12] provide an additional tool to probe the equation of state of dense nuclear and possibly quark matter [13-19] in the region of moderate temperatures and high baryon densities, close to those created in HIC.

First principle LQCD calculations suggest a smooth crossover transition at vanishing baryochemical potential $\mu_{B}=0$ from hadronic to partonic degrees of freedom [20]. Although there is no indication of a first- or a secondorder phase transition in the energy density, pressure, entropy density, and speed of sound, there are other observables in LQCD: chiral susceptibilities which seem to indicate a chiral crossover at a pseudocritical temperature $T_{\mathrm{pc}} \approx 155 \mathrm{MeV}$ $[21,22]$. However, the extension of LQCD calculations to 
finite $\mu_{B}$ is a difficult problem. Approximate lattice methods, such as extrapolations by Taylor expansion and the analytic continuation from purely imaginary to real $\mu_{B}$, are reasonable only for small baryon densities. The exploration of higher baryon densities requires effective QCD models, which respect the known symmetries of QCD and describe appropriately the known phenomenology of strong interactions.

The current knowledge of the properties of strongly interacting matter suggests a number of features that ought to be incorporated in any reasonable effective QCD model:

(i) First, nuclear matter and the nuclear liquid-vapor phase transition at moderate temperatures, close to the nuclear saturation density [23].

(ii) Second, at high temperature and at high chemical potential the Stefan-Boltzmann limit for massless quarks and gluons should be reached by thermodynamic quantities [24].

(iii) The transition from hadronic to quark-gluon degrees of freedom, at high temperatures and/or chemical potentials, is a crucial ingredient for the consistent description of QCD matter.

Often, these different aspects of QCD are modeled within separate frameworks, which are then merged through various constructions.

The present work formulates a single combined framework to describing the QCD thermodynamics, which simultaneously satisfies all the constraints from lattice QCD and known nuclear matter properties as well as neutron star observations.

The resulting equation of state is then used to estimate various properties of systems created in both heavy-ion collisions and neutron star physics. Section II presents the description of the chiral SU(3)-flavor parity-doublet Polyakov-loop quarkhadron mean-field model, CMF. Section III describes the fine tuning of model parameters to the $\mu_{B}=0 \mathrm{LQCD}$ data on the trace anomaly, and presents a comparison of the CMF model predictions for various conserved charge number fluctuations with the corresponding lattice data. The QCD phase diagram deduced from the CMF model is studied in Sec. IV. The creation of hot and dense QCD matter as created in heavyion collisions at various collision energies is studied using the one-dimensional hydrodynamics in Sec. V; the respective trajectories along the QCD phase diagram are explored as well. Section VI presents the CMF model predictions for the observed neutron star properties.

\section{CHIRAL SU(3)-FLAVOR PARITY-DOUBLET POLYAKOV-LOOP QUARK-HADRON MEAN-FIELD MODEL, CMF}

The chiral SU(3)-flavor parity-doublet Polyakov-loop quark-hadron mean-field model, $\mathrm{CMF}$, is an extension of the previously proposed $\sigma$ model with parity doubling for nuclear and hadron matter [25-30]. The CMF model was extended to include quark degrees of freedom [31-35]. This model is a phenomenological effective unified approach to describe interacting hadron-quark matter. The Lagrangian includes essential symmetries and features of QCD. These include (i) Chiral symmetry restoration in the hadronic sector, in particular the baryon parity doubling so an explicit mass term for baryons is possible, even when chiral symmetry is restored. This leads to a restoration of mass degeneracy among baryons and their respective parity partners $[36,37]$.

(ii) Eigenvolume corrections for hadrons, which allow for an effective modeling of their repulsive interactions. This suppresses hadronic densities and ensures a transition to a parton-dominated matter at large densities, when quark and gluon degrees of freedom appear.

(iii) Chiral symmetry restoration for quarks and a dynamical generation of their masses.

(iv) The Polyakov loop via a QCD-motivated potential, incorporates the deconfinement transition.

A detailed description of the hadronic part of the CMF model can be found in the literature [31,32,34,38]. It is based on a realization of a $\sigma$ model in the mean-field description. Here the relevant fermionic degrees of freedom are baryons that interact through mesonic mean fields. The version of this model used here includes all states in the $\mathrm{SU}(3)_{f}$ baryon octet, together with their parity partners, i.e., states with the same quantum numbers but opposite parity. The LQCD data suggests that the same mechanism should be implemented for higher baryonic states: the baryon decuplet [37]; this is a plan for future studies. In the limit of chiral symmetry restoration these parity partner states should be degenerate and their masses equal, which then serves as a signal for chiral symmetry restoration. To allow for such a behavior, the baryon masses are dynamically generated by the scalar $\sigma$ field and the scalar strange $\zeta$ field, which serve as the order parameters for the chiral transition:

$m_{i \pm}^{*}=\sqrt{\left[\left(g_{\sigma i}^{(1)} \sigma+g_{\zeta i}^{(1)} \zeta\right)^{2}+\left(m_{0}+n_{s} m_{s}\right)^{2}\right]} \pm g_{\sigma i}^{(2)} \sigma \pm g_{\zeta i}^{(2)} \zeta$.

Here + stands for positive and - for negative parity states, $g_{i}^{(j)}$ are the coupling constants of baryons to the two scalar fields, and $m_{0}=759 \mathrm{MeV}$ is the baryon mass at the restored phase. In addition, there is an $\mathrm{SU}(3)_{f}$ symmetry-breaking mass term proportional to the strangeness content of the baryons; there $n_{s}$ is the number of strange quarks in the baryon and $m_{s}=$ $130 \mathrm{MeV}$ is the mass of the strange quark. The couplings $g_{i}^{(j)}$ are tuned to reproduce the vacuum masses of baryons.

The mean-field values of the chiral fields are driven by the thermal contribution from baryons and quarks, and controlled by the scalar meson interaction, driving the spontaneous breaking of the chiral symmetry:

$$
V=V_{0}+\frac{1}{2} k_{0} I_{2}-k_{1} I_{2}^{2}-k_{2} I_{4}+k_{6} I_{6},
$$

with

$$
\begin{gathered}
I_{2}=\left(\sigma^{2}+\zeta^{2}\right), \quad I_{4}=-\left(\sigma^{4} / 2+\zeta^{4}\right), \\
I_{6}=\left(\sigma^{6}+4 \zeta^{6}\right)
\end{gathered}
$$

where $V_{0}$ is fixed by demanding that the potential vanishes in the vacuum.

The parameters of the scalar and the vector interactions are fitted to describe nuclear matter properties [34,39]. 
Contributions of all established hadronic resonances are included here with their vacuum masses [40]. These states can be coupled to meson fields as parity doublets as well. However, this is not done in the current implementation; they interact with the other particles via their excluded volume only.

The quark degrees of freedom are incorporated in accord with the Polyakov-loop extended Nambu-Jona-Lasinio (PNJL) approach [41]. The appearance of quarks is controlled by the value of Polyakov loop $L$ and its conjugate $L^{\dagger}$ which are matrices in color space. $L$ plays the role of the order parameter for the deconfinement transition. The coupling of the quarks to the Polyakov loop is introduced through the thermal energy of the quarks. Their thermal contribution to the grand canonical potential $\Omega$ is given by

$$
\Omega_{q}=-V T \sum_{i \in Q} \frac{d_{i}}{(2 \pi)^{3}} \int d^{3} k \frac{1}{N_{c}} \operatorname{Tr}_{\mathrm{c}} \ln \left(1+L e^{-\left(E_{i}^{*}-\mu_{i}^{*}\right) / T}\right)
$$

and

$$
\Omega_{\bar{q}}=-V T \sum_{i \in Q} \frac{d_{i}}{(2 \pi)^{3}} \int d^{3} k \frac{1}{N_{c}} \operatorname{Tr}_{\mathrm{c}} \ln \left(1+L^{\dagger} e^{-\left(E_{i}^{*}+\mu_{i}^{*}\right) / T}\right) .
$$

After carrying out the trace in color space, the Eqs. 4 and 5 can be formulated in terms of the traced Polyakov loop $\Phi=\left(\operatorname{Tr}_{\mathrm{c}} L\right) / N_{c}$ and $\bar{\Phi}=\left(\operatorname{Tr}_{\mathrm{c}} L^{\dagger}\right) / N_{c}$ [41]. At finite $\mu_{B}$ the Polyakov loop may become complex, which leads to the fermion sign problem [42-44]. In our calculations we impose $\Phi$ and $\bar{\Phi}$ to be real valued, so all thermodynamic quantities are real. At vanishing quark density $\Phi=\bar{\Phi}$, while at finite net quark density $\Phi \neq \bar{\Phi}$. The quark thermodynamic potential obtains the following form:

$$
\begin{aligned}
\Omega_{q}= & -V T \sum_{i \in Q} \frac{d_{i}}{(2 \pi)^{3}} \int d^{3} k \frac{1}{N_{c}} \ln \left(1+3 \Phi e^{-\left(E_{i}^{*}-\mu_{i}^{*}\right) / T}\right. \\
& \left.+3 \bar{\Phi} e^{-2\left(E_{i}^{*}-\mu_{i}^{*}\right) / T}+e^{-3\left(E_{i}^{*}-\mu_{i}^{*}\right) / T}\right)
\end{aligned}
$$

and

$$
\begin{aligned}
\Omega_{\bar{q}}= & -V T \sum_{i \in Q} \frac{d_{i}}{(2 \pi)^{3}} \int d^{3} k \frac{1}{N_{c}} \ln \left(1+3 \bar{\Phi} e^{-\left(E_{i}^{*}+\mu_{i}^{*}\right) / T}\right. \\
& \left.+3 \Phi e^{-2\left(E_{i}^{*}+\mu_{i}^{*}\right) / T}+e^{-3\left(E_{i}^{*}+\mu_{i}^{*}\right) / T}\right) .
\end{aligned}
$$

The sums run over all light quark flavors $(u, d$, and $s), V$ is the system's volume, $d_{i}=2 \times 3$ is the the corresponding degeneracy factor, and $E_{i}^{*}=\sqrt{m_{i}^{* 2}+p^{2}}$ is the energy. The quark chemical potential $\mu^{*}$ is defined by the quarks' quantum numbers and is not modified by repulsive interactions as those are disfavored by LQCD calculations $[39,45]$. Note that two- and three-quark contributions to $\Omega$ are omitted in the CMF model since hadronic excitations are explicitly included. The present model does not consider color superconductivity phenomena [46,47] and inhomogeneous phases [48].

The effective masses of the light quarks are generated by the $\sigma$ field (nonstrange chiral condensate) as well; the mass of the strange quark is generated by the $\zeta$ field (strange quark-antiquark state). The small explicit mass terms are $\delta m_{q}=5 \mathrm{MeV}$ and $\delta m_{s}=150 \mathrm{MeV}$ for the strange quark, and $m_{0 q}=253 \mathrm{MeV}$, which corresponds to an explicit mass term which does not originate from chiral symmetry breaking:

$$
\begin{aligned}
& m_{q}^{*}=-g_{q \sigma} \sigma+\delta m_{q}+m_{0 q}, \\
& m_{s}^{*}=-g_{s \zeta} \zeta+\delta m_{s}+m_{0 q} .
\end{aligned}
$$

Dynamics of the Polyakov loop is controlled by the effective Polyakov-loop potential $U_{\mathrm{Pol}}(\Phi, \bar{\Phi}, T)[41,49]$ :

$$
\begin{aligned}
U_{\mathrm{Pol}}(\Phi, \bar{\Phi}, T)= & -\frac{1}{2} a(T) \Phi \bar{\Phi}+b(T) \ln [1-6 \Phi \bar{\Phi} \\
& \left.+4\left(\Phi^{3}+\bar{\Phi}^{3}\right)-3(\Phi \bar{\Phi})^{2}\right], \\
a(T)= & a_{0} T^{4}+a_{1} T_{0} T^{3}+a_{2} T_{0}^{2} T^{2}, \quad b(T)=b_{3} T_{0}^{4} .
\end{aligned}
$$

Note there are other available expressions of the potential $[41,50,51]$. The parameters of the used potential can be fixed to the lattice QCD data in the pure gauge sector [51]. However, this yields an unsatisfactory description of the $(2+1)$ flavor QCD thermodynamics when the hadrons are explicitly included in the model. Therefore the parameters of the Polyakov-loop potential are adjusted in the present work to describe properly the $(2+1)$-flavor lattice data.

The CMF model incorporates excluded-volume (EV) effects, in order to suppress the hadronic degrees of freedom in the regions of the phase diagram where physically quarks and gluons dominate [32]. Consequently, all the thermodynamic densities, including the quark contribution, are reduced, as parts of the system are occupied by EV hadrons:

$$
\rho_{i}=\frac{\rho_{i}^{\mathrm{id}}\left(T, \mu_{i}^{*}-v_{i} p\right)}{1+\sum_{j \in \mathrm{HRG}} v_{j} \rho_{j}^{\mathrm{id}}\left(T, \mu_{j}^{*}-v_{j} p\right)},
$$

where $v_{j}$ are the eigenvolume parameters for the different species. $p$ is the system pressure and $\mu^{*}$ is the chemical potential of the hadron. The $v$ is assumed to be $v_{B}=1 \mathrm{fm}^{3}$ for (anti)baryons, $v_{M}=1 / 8 \mathrm{fm}^{3}$ for mesons, and is set to zero $v_{q}=0$ for quarks.

All in all, the CMF model has the following expression for the grand canonical potential:

$$
\Omega=\Omega_{q}+\Omega_{\bar{q}}+\sum_{i \in \mathrm{HRG}} \Omega_{i}-V\left(U_{\mathrm{sc}}+U_{\mathrm{vec}}+U_{\mathrm{Pol}}\right)
$$

where $\Omega_{q}$ and $\Omega_{\bar{q}}$ are quark and antiquark contributions given by Eqs. (6) and (7). $\sum_{i \in \mathrm{HRG}} \Omega_{i}$ represents the hadronic contribution and is given by

$$
\Omega_{i}=-V T \frac{d_{i}}{(2 \pi)^{3}} \int d^{3} k \ln \left[1+\eta_{i} \exp \left(-\frac{E_{i}^{*}-\mu_{i}^{*}+v_{i} p}{T}\right)\right],
$$

here and in Eqs. (6) and (7). $V$ is the system volume which is reduced by the presence of $\mathrm{EV}$ hadron resonance gas (HRG) hadrons as

$$
V=V^{\mathrm{id}}\left(1-\sum_{i \in \mathrm{HRG}} v_{i} \rho_{i}^{\mathrm{id}}\left(T, \mu_{i}^{*}-v_{i} p\right)\right) .
$$


For baryons $\eta_{i}=+1$ and for mesons $\eta_{i}=-1$. $v_{i}$, discussed above, is the EV parameter of the hadron. Octet baryons, $p, n, \Lambda, \Sigma^{+, 0,-}, \Xi^{0,-}$, and their respective parity partners, $N(1535)^{+, 0}, \Lambda(1405), \Sigma(1750)^{+, 0,-}, \Xi(1950)^{0,-}$, are a part of the HRG in the model, but also interact through mesonic fields, so their masses in $E_{i}=\sqrt{m_{i}^{* 2}+k^{2}}$ are calculated using Eq. (1) and their chemical potentials are shifted by vector fields:

$$
\mu_{i}^{*}=\mu_{i}-\left(g_{i \omega} \omega+g_{i \rho} \rho+g_{i \phi} \phi\right),
$$

where $\mu_{i}$ is the chemical potential with respect to the particles' charges. The rest of the HRG hadrons are assumed to have vacuum mass and are not coupled to vector fields.

The contribution of the mean field scalar mesons is

$$
U_{\mathrm{sc}}=\frac{1}{2} m_{\sigma}^{2} \sigma^{2}+\frac{1}{2} m_{\zeta}^{2} \zeta^{2}+\frac{1}{2} k_{0} I_{2}-k_{1} I_{2}^{2}-k_{2} I_{4}+k_{6} I_{6}+m_{\pi}^{2} f_{\pi} \sigma+\left(\sqrt{2} m_{\mathrm{K}}^{2} f_{\mathrm{K}}-\frac{1}{\sqrt{2}} m_{\pi}^{2} f_{\pi}\right) \zeta-k_{4} \ln \left(\frac{\sigma^{2} \zeta}{\sigma_{0}^{2} \zeta_{0}}\right)
$$

and the vector mesons' contributions are given by

$$
U_{\text {vector }}=\frac{1}{2} m_{\omega}^{2} \omega^{2}+\frac{1}{2} m_{\rho}^{2} \rho^{2}+\frac{1}{2} m_{\phi}^{2} \phi^{2}+g_{4}\left(\omega^{4}+\frac{1}{2} \rho^{4}+\frac{1}{4} \phi^{4}+3 \omega^{2} \rho^{2}+3 \omega^{2} \phi^{2}+\frac{4}{\sqrt{2}} \omega^{3} \phi+\frac{2}{\sqrt{2}} \omega \phi^{3}+\frac{3}{2} \rho^{2} \phi^{2}\right) .
$$

All of the following calculations are done in the meanfield approximation. Additionally the no-sea approximation [52] is used, so vacuum contributions from the Dirac sea are neglected. The CMF model contains a logarithmic term [Eq. (15)] similar to the one used in [53] which mimics the contribution of vacuum fluctuations to the thermodynamics. We also omit renormalization or quantum fluctuation effects here due to a lack of systematic knowledge in the range of temperatures and densities used.

\section{CONSTRAINING THE CMF MODEL TO THE LATTICE DATA}

To introduce the constraints on the CMF model parameters from lattice QCD at high temperature and zero net-baryon density we use the QCD trace anomaly $I$, "the interaction measure," as a reference:

$$
\frac{I}{T^{4}}=\frac{\varepsilon-3 P}{T^{4}} .
$$

Trace anomaly $I$ is assumed to depict the appearance of quark degrees of freedom. $I$ effectively reflects the change of number of degrees of freedom with an increase of temperature [54]. The hadronic models are capable of reproducing lattice data on $I$ for $T \lesssim 150 \mathrm{MeV}$ [32]. In this region $I$ reflects the increase of number of degrees of freedom due to the excitation of heavier resonances. However, the subsequent peak in $I$ and the following decrease is attributed to the appearance of quarks and gluons and so to the reduction of degrees of freedom. With this assumption the free parameters of the present model, namely parameters of the quark sector, are tuned to reproduce the lattice data on trace anomaly.

TABLE I. Best fit values of parameters extracted from a scan over the parameter space.

\begin{tabular}{lcccc}
\hline \hline$T_{0}(\mathrm{MeV})$ & $a_{1}$ & $a_{2}$ & $b_{3}$ & $g_{q \sigma}=g_{s \zeta}$ \\
\hline 180.0 & -11.67 & 9.33 & -0.53 & -1.0 \\
\hline \hline
\end{tabular}

The LQCD trace anomaly permits one to calculate all observable thermodynamic quantities. Analysis of other lattice data (chiral susceptibility) seems to show that for chirally related observables there is a crossover transition with a "pseudocritical" temperature at $T \approx 156 \mathrm{MeV}$. The analysis of this data by a phenomenological model suggests a halfhadron, half-quark composition in that region [55].

The parameters of the CMF model's quark sector needed to reproduce the trace anomaly data from LQCD are found by a least-mean-squares fitting procedure for the parameters of the Polyakov-loop potential $U_{\mathrm{Pol}}(\Phi, \bar{\Phi}, T)$ and for the coupling constants $g_{q \sigma}$ and $g_{s \zeta}$ of the quarks to the chiral condensates $\sigma$ and $\zeta$, respectively. All in all this fixes five model parameters: $T_{0}, a_{1}, a_{2}, b_{3}$, and $g_{q \sigma}=g_{s \zeta}$ (we set $g_{q \sigma}$ and $g_{s \zeta}$ to the same value). The quark parameter fitting is performed through a scan over the parameter space on a $8 \times 6 \times 7 \times 6 \times 6$ sized grid, minimizing the root-mean-square deviation of the CMF model data on $I / T^{4}$ from results computed on the lattice. The resulting parameter values are presented in Table I. A comparison of the CMF model to the lattice data is shown in Fig. 1.

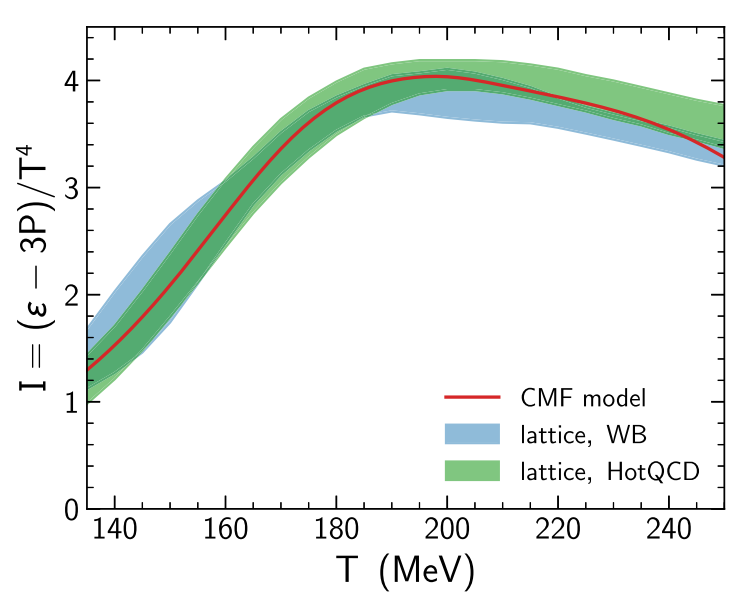

FIG. 1. Trace anomaly $I$ at $\mu_{B}=0$ as a function of temperature $T$. Comparison between model predictions and LQCD results $[21,22]$. 


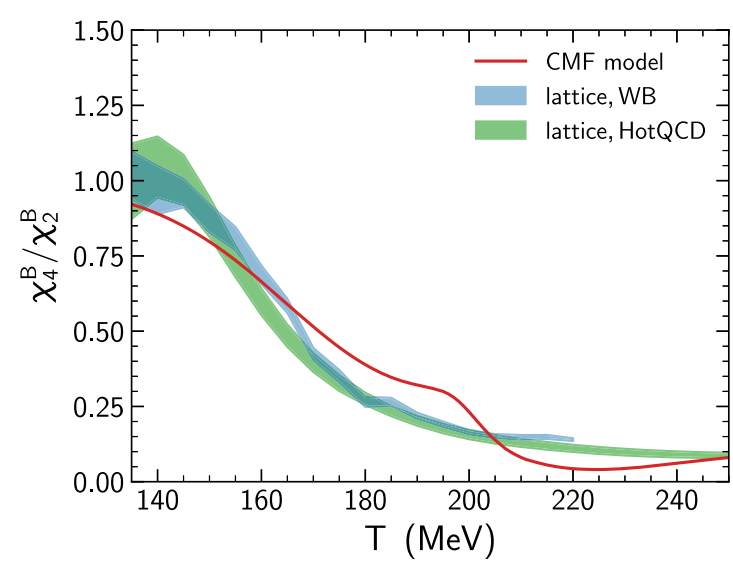

FIG. 2. The temperature dependence of net baryon number susceptibility ratio $\chi_{4}^{B} / \chi_{2}^{B}$ is shown as a function of temperature $T$. The red line depicts calculations within the CMF model, and blue and green color bands are the results of the lattice QCD calculations from the Wuppertal-Budapest and the HotQCD collaborations, respectively [56-59].

The values in Table I suggest couplings $g_{q \sigma}, g_{s \zeta}$ of quarks to the chiral fields of about $1 / 3$ of the baryons, which one may expect from the additive quark model. Larger values of quark couplings would significantly influence the size of the peak in interaction measure $I / T^{4}$, as studied in Ref. [34]. In the case of large values of $g_{q \sigma}, g_{s \zeta}$ the strong interplay between the chiral symmetry restoration and the deconfinement transition would result in too large values of the interaction measure. The large peaks in the baryon number susceptibilities are in contrast to the lattice data.

Higher order baryon number susceptibilities $\chi_{n}^{B}$ that are a LQCD measure of the particle number fluctuations,

$$
\chi_{n}^{B}=\frac{\partial^{n}\left(P / T^{4}\right)}{\left(\partial \mu_{B} / T\right)^{n}},
$$

as well as the curvatures of various lines of constant physical quantities are also interesting in the scope of LQCD data.

The behavior of $\chi_{4}^{B} / \chi_{2}^{B}$ at $\mu_{B}=0$ is presented in this section; the study of finite values of $\mu_{B}$ is presented in Sec. IV. A comparison of the CMF model with the available LQCD data for $\chi_{4}^{B} / \chi_{2}^{B}$ is shown in Fig. 2, indicating a fair agreement of the CMF model with the lattice data.

Lattice QCD studies often explore regions of finite $\mu_{B}$ by using the Taylor series expansion. The Taylor expansion in series of $T$ and $\mu_{B}$ up to $O\left(\mu_{B}^{4}\right)$ was used in [58] to calculate "lines of constant physics," i.e., lines in the $T-\mu_{B}$ plane where certain thermodynamic quantities like pressure, energy density, and entropy density, $P, \varepsilon, s$, are constant. The coefficients $\kappa_{2}^{f}$ and $\kappa_{4}^{f}(f \equiv P, \varepsilon, s)$ represent these contour lines in the $T-\mu_{B}$ plane using the following parametrization [58]:

$$
T_{f}\left(\mu_{B}\right)=T_{0}\left(1-\kappa_{2}^{f}\left(\frac{\mu_{B}}{T_{0}}\right)^{2}-\kappa_{4}^{f}\left(\frac{\mu_{B}}{T_{0}}\right)^{4}\right) .
$$

Here the coefficients $\kappa_{2}^{f}$ and $\kappa_{4}^{f}$ are calculated from Eqs. (20) and (21); see Ref. [58] for details:

$$
\kappa_{2}^{f}=\frac{1}{T_{0}} \frac{f_{2}\left(T_{0}\right)}{\left.\frac{\partial f_{0}(T)}{\partial T}\right|_{\left(T_{0}, 0\right)}}, \quad \kappa_{4}^{f}=\frac{\left.\frac{1}{2}\left(\kappa_{2}^{f}\right)^{2} T_{0}^{2} \frac{\partial^{2} f_{0}(T)}{\partial T^{2}}\right|_{\left(T_{0}, 0\right)}-\kappa_{2}^{f}\left(\left.T_{0} \frac{\partial f_{2}(T)}{\partial T}\right|_{\left(T_{0}, 0\right)}-2 f_{2}\left(T_{0}\right)\right)+f_{4}\left(T_{0}\right)}{\left.\frac{\partial f_{0}(T)}{\partial T}\right|_{\left(T_{0}, 0\right)}} .
$$

Here

$$
f_{2 n}(T)=\left.\frac{1}{(2 n) !} \frac{\partial^{2 n} f\left(T, \mu_{B}\right)}{\partial\left(\mu_{B} / T\right)^{2 n}}\right|_{(T, 0)} .
$$

The coefficients $\kappa_{2}^{f}$ and $\kappa_{4}^{f}$ are calculated in the CMF model for the pressure $P$, the energy density $\varepsilon$, and the entropy density $s$ as functions of the temperature $T$ (see Fig. 3 ). The CMF model predictions are in a reasonable agreement with recent LQCD calculations [58]. The rather low values of $\kappa_{2}^{f}$ and $\kappa_{4}^{f}$ suggest also small curvatures of lines of constant physical observables in the temperature region studied here. Effects of the finite chemical potential are small, therefore these lines are almost horizontal in the $T-\mu_{B}$ plane. The coefficients for the entropy and the energy density indicate that $\kappa_{2}^{s}<\kappa_{2}^{\varepsilon}$, meaning a decrease of the entropy density along the lines of constant energy density.

\section{THE CMF MODEL PHASE DIAGRAM}

Two order parameters, the chiral condensate $\sigma$ and the Polyakov loop $\Phi$, plus the interacting baryon octet within the $\mathrm{SU}(3)$-flavor $\sigma$ model permit four different phases within the CMF model. These phases are characterized as (i) A dilute gas of interacting hadrons.

(ii) A hadronic liquid: a dense hadronic phase, the transition from the hadron gas to the hadronic liquid is the nuclear liquid-vapor phase transition. Quarks start to appear in the hadronic liquid, but they are negligible.

(iii) A chirally restored phase, where the mass symmetry between the parity partners is restored. Here the quark masses are decreased, hence quarks give a sizable contribution to the thermodynamics.

(iv) A quark matter phase, where baryonic density is carried by quark degrees of freedom. The gluon contribution is modeled by the Polyakov-loop potential [51] and $\frac{1}{3} n_{q} / n_{B}=1$.

The baryon number susceptibilities $\chi_{n}^{B}$, that can be calculated using Eq. (18), are proportional to the respective cumulants of the baryon number distribution. Higher-order baryon number susceptibilities do increase in proportion to the increasing powers of the correlation length [60]. Such an 

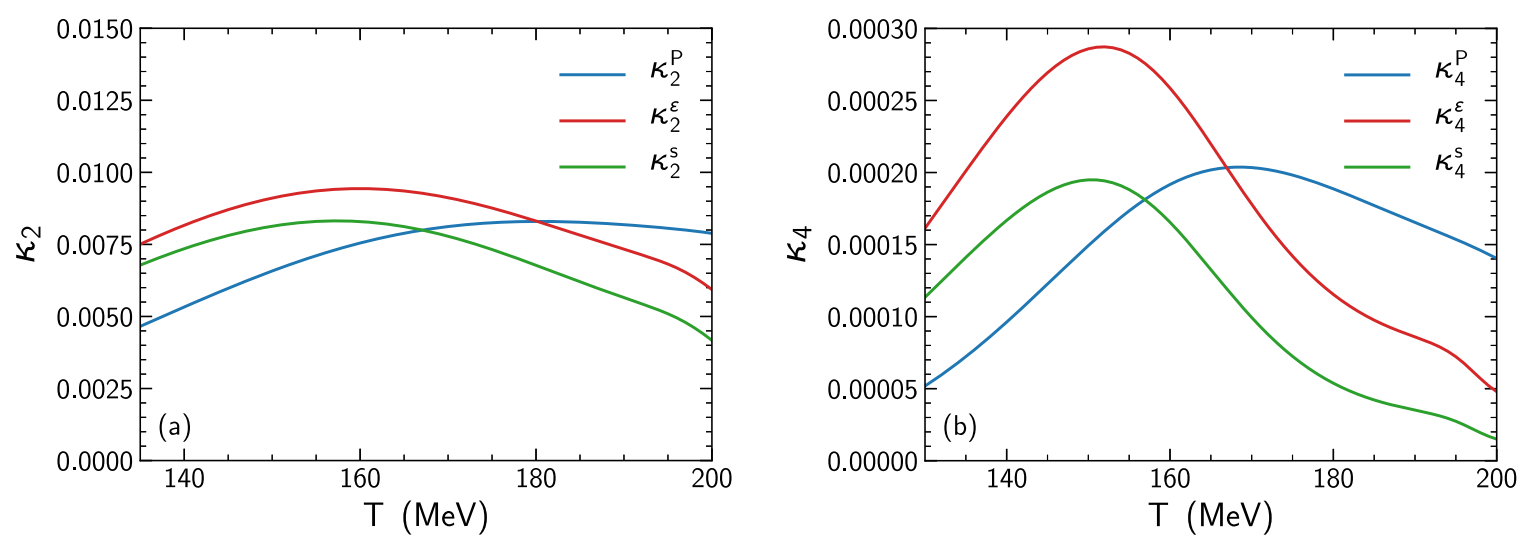

FIG. 3. Second $\kappa_{2}^{f}$ (a) and fourth $\kappa_{4}^{f}$ (b) coefficients of lines of constant pressure $P$, energy density $\varepsilon$, and entropy density $s$ versus temperature $T$. Explanation and lattice data can be found in [58].

increase in correlation length would be reflected in large values of second- and higher-order susceptibilities in the vicinity of a critical point and in the region of phase transition. Hence, these quantities are useful indicators of a critical behavior in the CMF model. Deviations of $\chi_{n}^{B}$ from the corresponding baselines indicate a transformation between different phases, which is reflected usually in a nonmonotonic behavior of these observables, e.g., skewness $\chi_{3}^{B} / \chi_{2}^{B}$ and kurtosis $\chi_{4}^{B} / \chi_{2}^{B}$.

The calculated skewness $\left(\chi_{3}^{B} / \chi_{2}^{B}\right)$ and kurtosis $\left(\chi_{4}^{B} / \chi_{2}^{B}\right)$ (Fig. 4) in the CMF model exhibit nontrivial structures in the $T-\mu_{B}$ phase diagram. The regions of deviations from baseline separate regions with quantitatively different properties, which are often dubbed "phases." Note that sharp phase boundaries indicate first-order phase transitions, FOPTs; these are only observable at quite moderate temperatures $T<$ $50 \mathrm{MeV}$. The hadron phase located at both low temperatures $T$ and baryon chemical potentials $\mu_{B}$ represents a dilute gas of interacting hadrons. There, the fluctuation measures $\chi_{2}^{B} / \chi_{1}^{B}$, $\chi_{3}^{B} / \chi_{2}^{B}, \chi_{4}^{B} / \chi_{2}^{B}$ are quite close to unity, consistent with the Skellam distribution baseline. The system exhibit a FOPT to a dense hadronic liquid phase with rising chemical potential $\mu_{B} \approx 1 \mathrm{GeV}$. Here, fluctuations are reduced due to the repulsive interactions. Quarks start to appear in moderation. The liquid phase exhibits an additional FOPT at $\mu_{B} \approx 1.5 \mathrm{GeV}$ and a second-order transition at $\mu_{B} \approx 2.4 \mathrm{GeV}$, which show up in the structure of the baryon number susceptibilities at these high $\mu_{B}$. The transition at $\mu_{B} \approx 1.5 \mathrm{GeV}$ is due to the restoration of chiral symmetry. The transition at $\mu_{B} \approx$ $2.4 \mathrm{GeV}$ is due to the quark matter phase where baryonic density is mainly contributed by quarks. The nonmonotonic behavior of the fluctuation measures $\chi_{3}^{B} / \chi_{2}^{B}$ and $\chi_{4}^{B} / \chi_{2}^{B}$ reflect the transitions. In contrast to the liquid-vapor transition, those two transitions do not change the Skellam baseline. Hence, the fluctuation measures are rather small, $\lesssim 1$, before and after the "transition."

The chiral critical point $(\mathrm{CP})$ of the CMF model is located at a rather low temperature $T_{\text {chiral }}^{\mathrm{CP}} \approx 17 \mathrm{MeV}$. This value is close to the critical temperature of the nuclear liquid-gas transition in the same model; the critical $\mu_{B}^{\mathrm{CP}}$ is remarkably different, though. The appearance of the parity partners controls the dynamics of the chiral fields: as the parity partners-in the CMF model—obey the same repulsive interaction strength as the nucleons, the critical point appears at that low temperature. This phenomenon has been observed in various mean-field models before.

The different phases shown in Fig. 5 in the $T-\mu_{B}$ plane are related to the chiral field $\sigma$ and the quark fraction. The chiral field is close to its vacuum value, $\sigma=\sigma_{0}$, in the hadron gas region; here the quark fraction is close to zero, as expected. Both observables deviate from their vacuum values at higher densities and temperatures only.
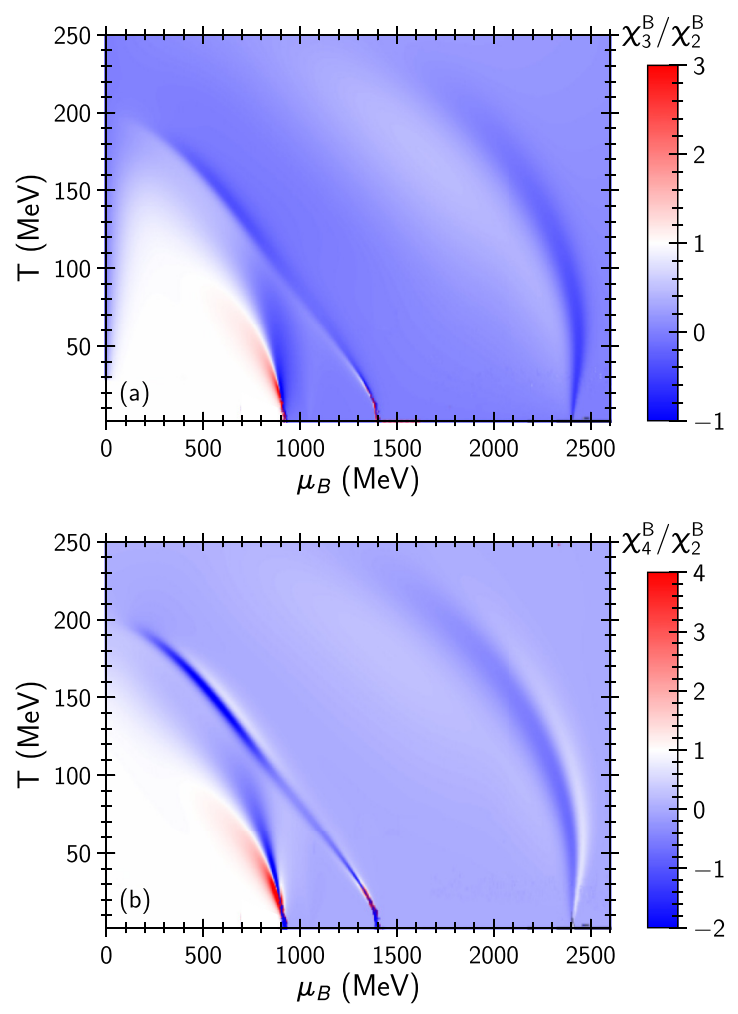

FIG. 4. Ratios of the CMF baryon number susceptibilities: $\chi_{3}^{B} / \chi_{2}^{B}$ skewness (a) and $\chi_{4}^{B} / \chi_{2}^{B}$ kurtosis (b) in the plane of baryon chemical potential $\mu_{B}$ and temperature $T$. Note the 3 distinct critical regions, with their remnants reaching from $T=0$ up to $T>$ $200 \mathrm{MeV}$. Note the absence of calculation results for skewness at $\mu_{B} \approx 0$ and $T<100 \mathrm{MeV}$ region. 

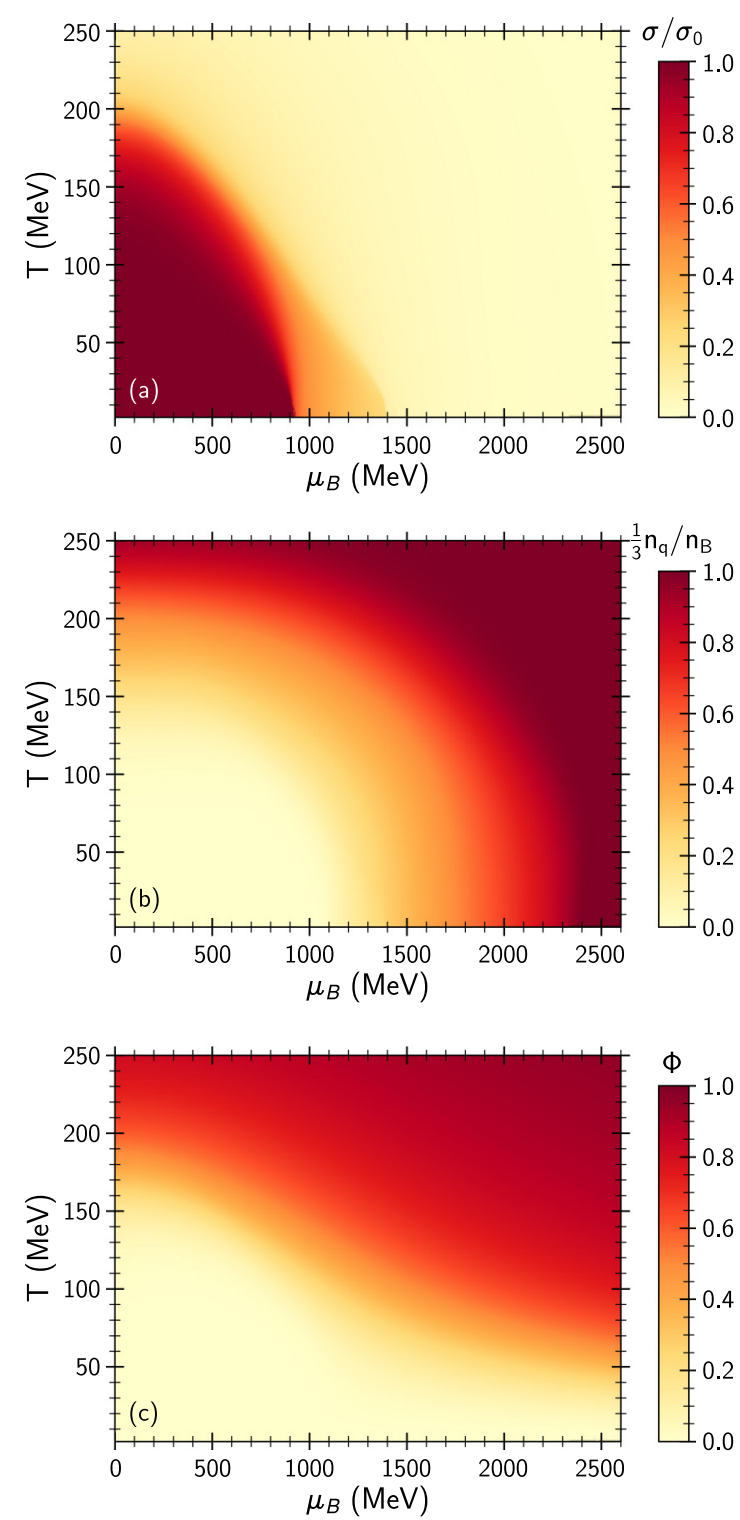

FIG. 5. The normalized nonstrange chiral condensate $\sigma / \sigma_{0}$ (the sigma field) (a), the quark fraction $\frac{1}{3} n_{q} / n_{B}$ (b), and the value of Polyakov loop $\Phi$ (c) of the CMF model in the plane of baryon chemical potential $\mu_{B}$ and temperature $T$. Note that the rather fast change of the chiral condensate appears at moderate energy densities, while deconfinement appears only at much higher energy densities and/or chemical potentials.

The chiral field drops off more slowly for $\mu_{B}=0$ than seen in lattice QCD calculations, where the chiral field rapidly drops around $T=155 \mathrm{MeV}$. The reason for this discrepancy is due to the fact that in the present CMF model the thermodynamics at these temperatures are strongly influenced by baryonic resonances which are not coupled to the chiral fields. Baryon resonances like the $\Delta$ ought to be coupled to chiral fields including their chiral partners. This brings down the chiral condensate to lower temperature, as can be seen in [61].

The speed of sound is another important signature for simulations of the dynamics of heavy-ion collisions and neutron star mergers. $c_{s / n_{B}}^{2}$ presents a derivative $c_{s / n_{B}}^{2}=\frac{\partial P}{\partial \varepsilon}$ at

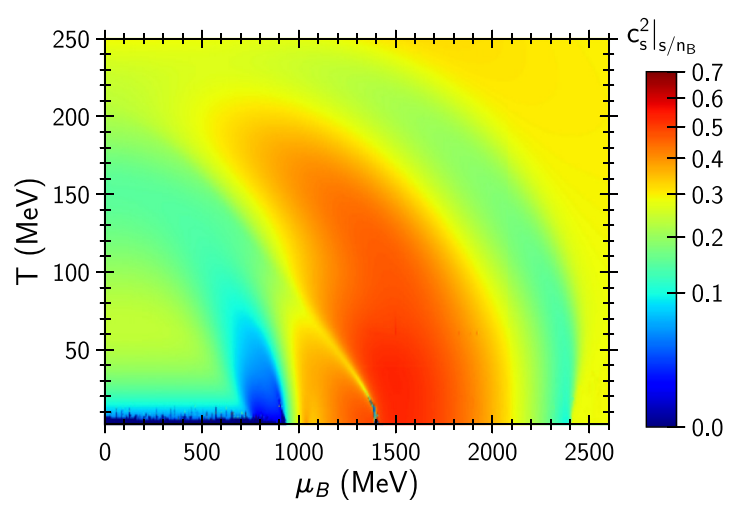

FIG. 6. The CMF model results for the square of the isentropic speed of sound, $c_{s}^{2}=\left(\frac{\partial p}{\partial \epsilon}\right)_{s / n_{B}}$, calculated along lines of constant total entropy density per net baryon density, $s / n_{B}=$ const, from Eq. (22).

constant $S / A=s / n_{B}$ entropy per baryon, that allows one to estimate a speed of propagation of sound-like excitations in nondissipative hydrodynamic evolution. The isentropic speed of sound can be calculated as [62]

$$
c_{s / n_{B}}^{2}=\frac{n_{B}^{2} \partial_{T} s-2 n_{B} s \partial_{\mu_{B}} s+s^{2} \partial_{\mu_{B}} n_{B}}{(\epsilon+P)\left(\partial_{\mu_{B}} n_{B} \partial_{T} s-\left(\partial_{T} n_{B}\right)^{2}\right)} .
$$

The partial derivatives with respect to the chemical potential and to the temperature are performed at constant temperature and at constant chemical potential, respectively. The calculated speed of sound shows three local minima which correspond to three locally softest points of the equation of state $(\mathrm{EoS})$; see Fig. 6. These three minima correspond to phase boundaries, where the baryon number susceptibilities present a nonmonotonic behavior. Note that the speed of sound reaches quite large values, $c_{s}^{2} \approx 0.7$, in the higher density region of nuclear matter. This high speed of sound is due to the strong repulsion between the baryons, before the onset of deconfinement. Thereafter, the vector repulsion and baryon excluded volume cease, as such terms have not been predicted for the quarks $[39,45]$.

\section{APPLICATION TO HEAVY-ION COLLISIONS}

The presented EoS is used as input for hydrodynamical simulations of both heavy-ion collisions and neutron star mergers. To illustrate which regions of the phase diagram can be reached in collisions at low and moderate collision energies, the stationary one-dimensional Taub adiabat model is used [63-65]. The expansion is described at lines of constant entropy per baryon, $S / A=$ const (isentropes). These lines depict the isentropic matter evolution of ideal fluid dynamics at different collision energies.

The entropy is produced in the earliest stage of a heavy-ion collision by the shock of violent compression [66]. During the system's expansion there is only a moderate increase of entropy due to the rather small viscosity $[67,68]$, hence, an isentropic expansion scenario is a reasonable approximation [69].

The expansion of the equilibrated matter then continues until the system becomes so dilute that the chemical as well 
as the kinetic freeze-out occur and the chemical composition is fixed.

The entropy per baryon $(S / A)$ is calculated in the onedimensional stationary scenario of central heavy-ion collisions: the two colliding slabs of cold nuclear matter $[65,66,70-74]$ conserve the baryon number, energy, and momentum across the shock front in accord with the relativistic Rankine-Hugoniot equation (Taub adiabat), RRHT [63,64]. Thus, the produced entropy is directly associated to the collision energy. The thermodynamic properties across the shock front are described by the RRHT equation

$$
\left(P_{0}+\varepsilon_{0}\right)\left(P+\varepsilon_{0}\right) n^{2}=\left(P_{0}+\varepsilon\right)(P+\varepsilon) n_{0}^{2},
$$

where $P_{0}, \varepsilon_{0}$, and $n_{0}$ correspond to the initial pressure, energy density, and baryon density in the local rest frame of each of the two slabs. The two symmetric slabs consist of the nuclear matter in the ground state, $P_{0}=0, \varepsilon_{0} / n_{0}-m_{N}=-16 \mathrm{MeV}$, and $n_{0}=0.16 \mathrm{fm}^{-3}$. With any known relation $P=P(\varepsilon, n)$, Eq. (23) can be solved. Furthermore, the collision energy is related to the created density as follows:

$$
\gamma^{\mathrm{CM}}=\frac{\varepsilon n_{0}}{\varepsilon_{0} n}, \quad \gamma^{\mathrm{CM}}=\sqrt{\frac{1}{2}\left(1+\frac{E_{\mathrm{lab}}}{m_{N}}\right)} .
$$

Here $\gamma^{\mathrm{CM}}$ is the Lorentz gamma factor in the center-of-mass frame of the heavy-ion collisions and $E_{\text {lab }}$ is the beam energy per nucleon in the laboratory frame of a fixed target collision. This relation can be obtained from the full stopping condition [65,66,71-75]. The initial state thermodynamics (density, temperature, and entropy) of the hot, dense participant matter is obtained from Eqs. (23) and (24) as a function of the collision energy. The known initial entropy yields the lines of constant entropy which give the trajectories of the heavy-ion collisions in the phase diagram.

The predicted isentropic expansion trajectories are shown in the $T-\mu_{B}$ plane phase diagram in Fig. 7.

Note that one-dimensional (1D) stationary RRHT-adiabat scenario predicts a very strong compression and heating already at intermediate laboratory (fixed target) bombarding energies. The heavy-ion participant system crosses the weak chiral transition predicted by the present CMF model already at $E_{\text {lab }} \approx 2 \mathrm{~A} \mathrm{GeV}$, i.e., at GSI's SIS18 accelerator facility. Here the specific total entropy is predicted to reach $S / A \approx 3$, in accord with previous RMF calculations [74] which also used the 1D RRHT-scenario. The $T-\mu_{B}$ values, $T \approx 70 \mathrm{MeV}$, $\mu_{B} \approx 1.2 \mathrm{GeV}$, with net baryon densities $n_{B} / n_{0} \approx 3$, reached here in heavy-ion collisions, coincide with the $T-\mu_{B}$ values reached in binary neutron star collisions, as recent general relativistic fully $3+1$-dimensional hydrodynamical calculations have confirmed $[16,18]$ for the gravitational wave event GW170817. At these temperatures and densities, $T \approx 70$ $\mathrm{MeV}$ and $n_{B} / n_{0} \approx 3$, the RRHT model predicts that about $20 \%$ of the dense matter has already transformed to quarks.

At $E_{\mathrm{lab}}=5.6 \mathrm{~A} \mathrm{GeV}$ and $\sqrt{s_{N N}}=3.5 \mathrm{~A} \mathrm{GeV}$ roughly $40 \%$ of the CMF matter is in the quark state in the RRHT model, which is a prerequisite for hot quarkyonic matter. Hence, this energy, which is presumably only reachable by the BMN detector at the Nuclotron at JINR Dubna and by the FXT fixed target setup at the STAR detector at RHIC, is of great interest:

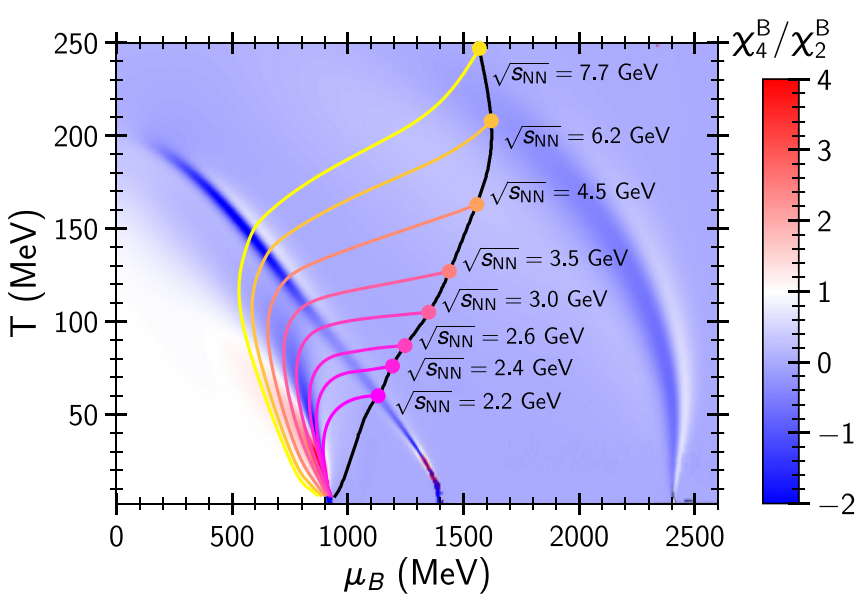

FIG. 7. Evolution of heavy-ion collisions in the high baryon density region of the $T-\mu_{B}$ phase diagram for different collision energies. Black line: Taub adiabat which describes the initial state of heavy ion collisions as an implicit function of $\sqrt{s_{N N}}$. Colored lines: isentropic lines of constant entropy per baryon $S / A$ at different bombarding energies $\sqrt{s_{N N}}$. See Table II for details.

here the matter starts to be dominated by quarks, rather then by in-medium baryons, at $T>100 \mathrm{MeV}$ and $\mu_{B} \gtrsim 1.5 \mathrm{GeV}$. This is predicted by the present CMF model, when using the 1D RRHT ideal hydrodynamics. This model predicts that the quarkyonic transition is crossed also at higher energies, using the isentropic expansion of the matter at specific total entropy $S / A>6$. In fact, nonequilibrium viscous effects may increase the specific entropy of the system. However, prefreeze-out radiation, e.g., of kaons and other hadrons with small scattering crosssections which can escape early from the semiequilibrated baryon-rich, dense system, can considerably lower the specific entropy during the expansion. So an answer to the question of whether the local entropy per baryon increases or decreases during the time evolution awaits more detailed microscopic and macroscopic modeling.

Hence, heavy ion fixed target experiments using SIS at FAiR and SPS at CERN as well as the STAR BES program at RHIC probe temperatures $50<T<280 \mathrm{MeV}$ and chemical potentials $500<\mu_{B}<1700 \mathrm{MeV}$ for the collision energy

TABLE II. Initial state properties obtained from the onedimensional stationary case of the central heavy-ion collision: Taub adiabat $[63,64]$. The entropy per baryon $S / A$, temperature $T$, the initial baryon density $n_{B} / n_{0}$, and the quark fraction $\frac{1}{3} n_{q} / n_{B}$ are presented for various colliding energies.

\begin{tabular}{lcrrrr}
\hline \hline$\sqrt{s_{N N}}(\mathrm{GeV})$ & $E_{\text {lab }}(\mathrm{GeV})$ & $S / A$ & $T(\mathrm{MeV})$ & $n_{B} / n_{0}$ & $\frac{1}{3} n_{q} / n_{B}$ \\
\hline 2.2 & 1.6 & 2.8 & 60.0 & 2.8 & 0.11 \\
2.4 & 2.1 & 3.5 & 76.0 & 3.4 & 0.20 \\
2.6 & 2.7 & 4.1 & 87.0 & 3.9 & 0.26 \\
3.0 & 3.9 & 5.3 & 105.0 & 4.9 & 0.35 \\
3.5 & 5.6 & 6.6 & 127.0 & 6.0 & 0.46 \\
4.5 & 9.9 & 8.4 & 163.0 & 8.6 & 0.70 \\
6.2 & 19.6 & 10.7 & 208.0 & 14.8 & 0.96 \\
7.7 & 30.7 & 13.1 & 247.0 & 18.7 & 1.00 \\
\hline \hline
\end{tabular}



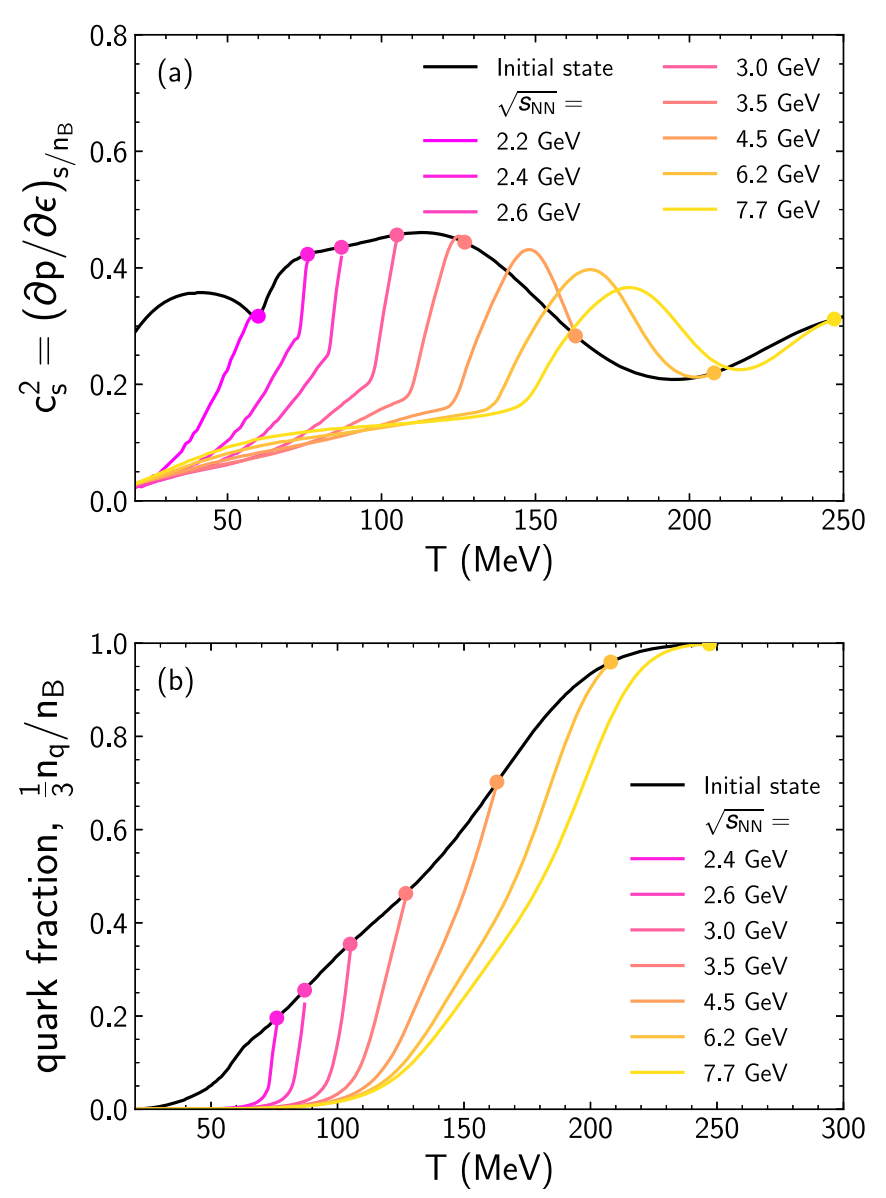

FIG. 8. Speed of sound squared $c_{s}^{2}$ at constant entropy per baryon (a) and quark fraction (b) along the isentropes as functions of temperature $T$. Colored lines correspond to different collision energies (initial entropy per baryon $S / A$ ); black solid lines correspond to the initial speed of sound and the quark fraction respectively. Isentropes are the same as in Fig. 7. See Table II for details.

range $\sqrt{s_{N N}}<10 \mathrm{GeV}$ considered here. In this region the CMF model shows not an additional phase transition, but the remnants of the nuclear liquid-vapor transition at $T \approx$ $20 \mathrm{MeV}$. The chiral transition at larger chemical potentials can influence the dynamical evolution, too. The present results suggest that heavy-ion collisions mostly probe regions where the nuclear matter liquid-vapor critical point dominates; hence, the observed baryon fluctuations are largely due to remnants of the nuclear liquid-vapor phase transition. This had been suggested also in previous works [34,76-78]. The $\mathrm{CP}$ associated with the chiral symmetry restoration in the CMF model lies at $\mu_{B} \approx 1.5 \mathrm{GeV}$ and $T \approx 17 \mathrm{MeV}$. This high density region is to the best of our knowledge is reachable in neutron star (NS) interiors and in binary general relativistic NS mergers $[16,17,19,79,80]$.

Figure 8 presents the square of the isentropic speed of sound at fixed specific entropy, $c_{s}^{2}$, and the quark fraction as function of the temperature for the studied collision energies; i.e., it shows how respective observable quantities evolve during the cooling of the system while it expands. Those isentropic lines which belong to $\sqrt{s_{N N}}>4.5 \mathrm{GeV}$ probe the softest point of the EoS, which is attributed to the chiral symmetry restoration. At this energy region there are strong local maxima and minima of the speed of sound squared, after which $c_{s}^{2}$ rapidly increases during the expansion due to the decrease of the quark fraction; as a result of the rapid appearance of baryons, the EoS stiffens quickly due to the hard-core baryon-baryon repulsion. For collision energies $\sqrt{s_{N N}}<4.5 \mathrm{GeV}$ the initial state is not dominated by quarks, hence the system starts to expand at rather high values of $c_{s}^{2}$, which then monotonically decrease during the expansion, as a result of the diminishing repulsion between the baryons.

\section{APPLICATION TO NEUTRON STARS}

The densities in neutron star interiors also can exceed by several times the nuclear matter saturation density. At these high densities, the lack of detailed knowledge of the equation of state and the appropriate microscopic degrees of freedom is similarly disturbing as in the relativistic heavy-ion collisions discussed above. The discussion of the role of hyperonic, quarkyonic, and strange quark degrees of freedom at these NS densities is ongoing.

The CMF model can be employed directly to describe neutron star matter. Here we work without any changes to the coupling constants and parameters used to describe the $\mu_{B}=0$ LQCD results. The temperatures isolated in neutron star interiors are negligibly small, in comparison to what we observe in heavy ion collisions and hot QCD scales. The calculations here are done in the limit $T=0$. In contrast to ordinary isospin symmetric nuclear matter, the neutron star matter is in $\beta$ equilibrium, which preserves the total electric neutrality of the NS matter and locally ensures stability with respect to $\beta$ decay. As a consequence, strangeness and hypercharges assume finite nonzero values. These constraints require the presence of leptons. In addition, one must allow for quark-hadron degrees of freedom. At $T=0$ quarks naturally appear due to the deconfinement mechanism of the Polyakov-loop order parameter. In this case $\Phi=\bar{\Phi}=0$ and only last term in (6) survives, because the model reduces to the NJL one.

In the case when two conserved charges are present, namely, electric and baryonic, a phase transition is noncongruent [81-83] as a result of global charge conservation. The CMF model predicts only one, chiral, phase transition for matter in $\beta$ equilibrium. This transition is noncongruent and results only in moderate increase of baryon density between two phases.

Figure 9 depicts the CMF model predictions of the relative particle densities of all different particle species present inside a CMF neutron star, at $T=0$, as a function of baryochemical potential. One feature of the present CMF model is the absence of baryon resonances (deltas, etc., and those of hyperons) even though they are included in the CMF model. Their total absence in the present CMF model calculation at $T=0$ is due to the very strong hard-core repulsion by the excluded volume corrections.

The calculated EoS at $T=0$ can be used as the input for the Tolman-Oppenheimer-Volkoff (TOV) equation, which allows one to relate the mass and the radius of any static, 


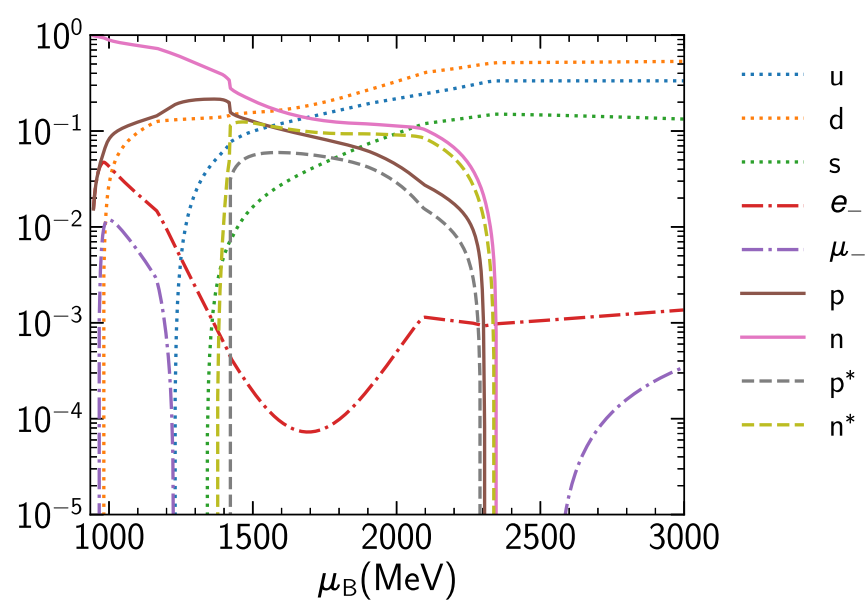

FIG. 9. The particle densities, normalized to the baryon density $n_{i} / n_{B}$, where a factor of $1 / 3$ is used for quarks, are shown as functions of baryon chemical potential $\mu_{B}$. Dotted lines: quarks; dashdotted lines: leptons; solid lines: nucleons; dashed lines: nucleon parity partners $p^{*}$ and $n^{*}$. The CMF calculations are performed in $\beta$ equilibrium at $T=0$.

spherical, gravitationally bound object $[84,85]$, i.e., here a static neutron star. The outer layer of a neutron star presumably consist of mostly neutron rich nuclei and clusters in chemical and $\beta$ equilibrium. Those nuclei are not yet included in the CMF model. Hence, another input for the EoS of the NS crust is needed. Here, we use the classical crust EoS [86] matched to the CMF EoS at $n_{B} \approx 0.05 \mathrm{fm}^{-3}$.

Figure 10 presents the results on the NS mass-radius relations obtained by solving the TOV equation with the present CMF EoS, matched just to that crust EoS. The total fraction of the star's mass which consists of light and strange quarks is presented in color code. The most massive stable solution of the TOV equation contains only $<30 \%$ deconfined quarks; i.e., for lighter NSs only a small fraction of the star's mass originates from deconfined quark matter. If the quark fraction is increased above $30 \%$ the stars become unstable. The central density of the stable stars can never exceed $n_{B}=6 n_{0}$, as shown in the lower part of Fig. 10. Here again the maximum mass indicates the "last stable star." The continuous slow transition from NS matter to a sizable deconfined quark phase implies a smooth appearance of quarks in the star structure. This does prohibit a strict separation between a quark core and the hadronic interior of the star. This is a CMF result due to the Polyakov-loop implementation of the deconfinement mechanism and no vector repulsion among quarks. The absence of quark repulsion along with the smooth appearance of quarks disfavors a "second family" of neutron stars since in the quark phase EoS is soft and cannot support strong gravitational compression. Though LQCD data disfavors repulsive forces for quarks, there is active discussion in the astrophysical community on the role of the vector repulsion in the physics of neutron stars [87-89]. An approach with density dependent quark vector coupling is developed in [90] where it is argued that the repulsion arises from nonperturbative gluon exchange.

Results similar to ours are obtained in the quarkyonic matter model, where the deconfinement is realized by the
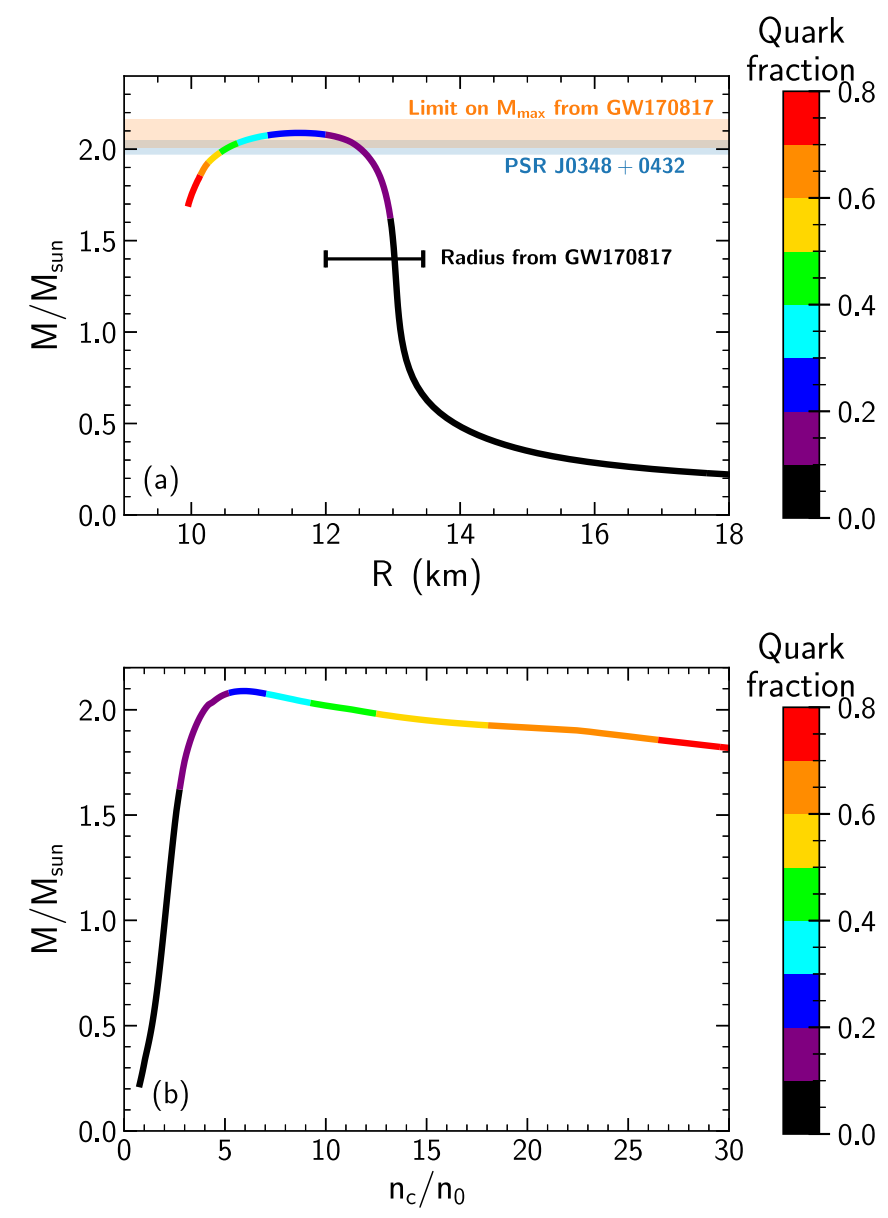

FIG. 10. The CMF mass-radius diagram (a) for neutron stars is shown as calculated within the CMF model. The CMF neutron star mass is shown as a function of central density $n_{c}$ normalized to saturation density $n_{0}(\mathrm{~b})$. Color indicates the fraction of the star's mass due to quarks.

appearance of quarks from inside of the Fermi sea while the hadrons there reside exclusively on the surface shell in momentum space [1]. A similar approach to deconfinement was suggested in $[91,92]$; however, there the produced massradius diagram differs from the CMF model [35] due to the different realization of the chiral symmetry restoration.

The response of a neutron star to nonspherical gravitational fields is reflected in the tidal deformability coefficient $\lambda$ [93], which depends strongly on the EoS. During the inspiral phase of a binary neutron star merger, both neutron stars experience tidal deformations induced by the accompanying neutron star partner. The tidal deformability $\lambda$ is a measure of the induced quadruple moment $Q_{i j}$ in response to the external tidal field $\mathcal{E}_{i j}$ :

$$
Q_{i j}=-\lambda \mathcal{E}_{i j}
$$

$\lambda$ is directly proportional to the second Love number $k_{2}$ :

$$
\lambda=\frac{2}{3} k_{2} R^{5}
$$



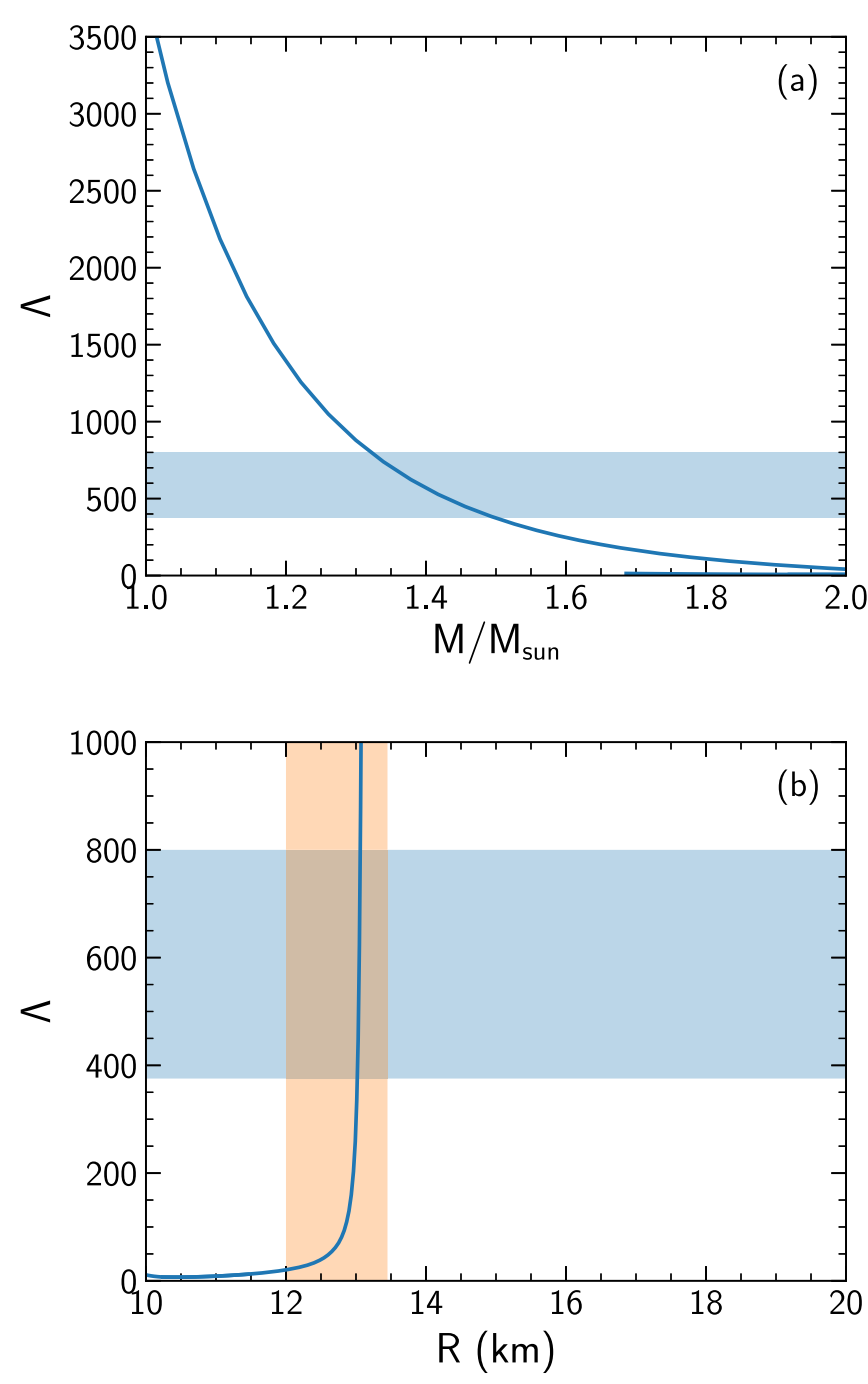

FIG. 11. Tidal deformability $\Lambda$ as function of NS mass (a) and radii (b). Blue bands correspond to $\Lambda$ constraints of a NS with $M=$ $1.4 M_{\text {sun }}$, and yellow bands correspond to constraints on the radius of a NS with $M=1.4 M_{\text {sun }}[14]$.

For convenience, usually the dimensionless tidal deformability $\Lambda$ is presented as

$$
\Lambda=\frac{\lambda}{M^{5}}=\frac{2}{3} k_{2}\left(\frac{R}{M}\right)^{5} .
$$

Here, $M$ and $R$ are the mass and radius of the neutron star. A proper value of $\Lambda$ is important for the description of the inspiral stage during the merger of two neutron stars.

Various estimates of $\Lambda$ emerged after the detection of GW170817 by the LIGO and the Virgo collaborations [12]. Reference [13] argued that for a $1.4 M_{\odot}$ neutron star the tidal deformability and star radius are constrained to $\Lambda_{1.4 M_{\odot}}>$ 120 and $R_{1.4 M_{\odot}}<13.6 \mathrm{~km}$. It was concluded by means of a Bayesian analysis that for a $1.4 M_{\odot}$ star the deformability should be $375.5<\Lambda_{1.4 M_{\odot}}<800$ and the radius is at $12.00<$ $R_{1.4 M_{\odot}}<13.45 \mathrm{~km}$, with respective $2 \sigma$ confidence levels; see Ref. [14] and Fig. 11. A recent analysis by the LIGO and
Virgo collaborations [15,94] provides detailed constraints, by using a Bayesian analysis based on reproducing the details of the gravitational wave signal.

\section{SUMMARY}

A unified and consistent approach to QCD thermodynamics, which is appropriate for essentially all temperatures and densities relevant for both heavy-ion collisions and for neutron star matter, is presented. The chiral SU(3)-flavor paritydoublet Polyakov-loop quark-hadron mean-field model, or CMF model, includes the main features of QCD hadron phenomenology, as well as a good description of known QCD thermodynamics. The CMF model allows for a simultaneous description of many nuclear (astro)physical data, consistent with astrophysical observations of compact stars as well as heavy-ion collisions. The CMF model is improved by fixing the relevant CMF parameters for the quark sector to the state-of-the-art LQCD data on the interaction measure. Here, the parameters of the Polyakov-loop potential and of the quark couplings to the chiral fields have been fixed. A good agreement is found between the CMF model predictions for LQCD data on both the baryon number susceptibilities and the "lines of constant physics." The CMF model is used to explore the phase diagram of strongly interacting matter for a wide range of $T$ and $\mu_{B}$. Three critical regions are found, which are connected to the nuclear liquid-vapor phase transition, to the chiral symmetry restoration, and to the quark matter. The model predicts two critical points: one associated with a nuclear liquid-vapor phase transition with critical temperature $T_{\mathrm{CP}}^{\text {nucl }} \approx 20 \mathrm{MeV}$ and one from chiral symmetry restoration with $T_{\text {chiral }}^{\mathrm{CP}} \approx 17 \mathrm{MeV}$. The transition to quark matter is always a smooth crossover. The region of the phase diagram accessible to experiments of high energy heavy-ions collisions is dominated by remnants of the nuclear liquid-vapor phase transition. Other critical regions may be probed by neutron star structure and binary neutron star mergers. The calculated properties of neutron stars, such as the mass-radius relation, the chemical composition, and the tidal deformability, are in a good agreement with recent experimental observations. The applicability of the improved CMF model to such a wide range of strongly interacting systems is impressive. For the first time, a QCD-motivated EoS is presented which precisely describes the thermodynamic observables for the whole QCD phase diagram.

\section{ACKNOWLEDGMENTS}

The authors thank HIC for FAIR and HGS-HIRe for FAIR. This work was supported by the DAAD through a PPP exchange grant. J.S. thanks the BMBF through the ErUMData project for funding and acknowledges the support of the SAMSON AG, WGG-Forderverein, and the C.W. FückStiftungs Prize 2018. H.S. acknowledges support through the Judah M. Eisenberg Laureatus Chair at Goethe University and the Walter Greiner Gesellschaft, Frankfurt. Computational resources have been provided by the Center for Scientific Computing (CSC) at the J. W. Goethe-University, Frankfurt. 
[1] L. McLerran and S. Reddy, Phys. Rev. Lett. 122, 122701 (2019).

[2] A. Kurkela, P. Romatschke, and A. Vuorinen, Phys. Rev. D 81, 105021 (2010).

[3] E. V. Shuryak, Phys. Rep. 61, 71 (1980).

[4] K. Rajagopal, Nucl. Phys. A 661, 150 (1999).

[5] M. G. Alford, Nucl. Phys. Proc. Suppl. 117, 65 (2003).

[6] M. Buballa, Phys. Rep. 407, 205 (2005).

[7] T. Schafer arXiv:hep-ph/0304281.

[8] K. Fukushima and T. Hatsuda, Rep. Prog. Phys. 74, 014001 (2011).

[9] K. Takami, L. Rezzolla, and L. Baiotti, J. Phys.: Conf. Ser. 600, 012056 (2015).

[10] E. Braaten and A. Nieto, Phys. Rev. D 53, 3421 (1996).

[11] P. de Forcrand, PoS LAT2009, 010 (2009).

[12] B. Abbott et al. (LIGO Scientific Collaboration and Virgo Collaboration), Phys. Rev. Lett. 119, 161101 (2017).

[13] E. Annala, T. Gorda, A. Kurkela, and A. Vuorinen, Phys. Rev. Lett. 120, 172703 (2018).

[14] E. R. Most, L. R. Weih, L. Rezzolla, and J. Schaffner-Bielich, Phys. Rev. Lett. 120, 261103 (2018).

[15] B. P. Abbott et al. (LIGO Scientific Collaboration and Virgo Collaboration), Phys. Rev. Lett. 121, 161101 (2018).

[16] E. R. Most, L. J. Papenfort, V. Dexheimer, M. Hanauske, S. Schramm, H. Stöcker, and L. Rezzolla, Phys. Rev. Lett. 122, 061101 (2019).

[17] A. Bauswein, N.-U. F. Bastian, D. B. Blaschke, K. Chatziioannou, J. A. Clark, T. Fischer, and M. Oertel, Phys. Rev. Lett. 122, 061102 (2019).

[18] M. Hanauske, J. Steinheimer, L. Bovard, A. Mukherjee, S. Schramm, K. Takami, J. Papenfort, N. Wechselberger, L. Rezzolla, and H. Stoecker, J. Phys.: Conf. Ser. 878, 012031 (2017).

[19] M. Hanauske, J. Steinheimer, A. Motornenko, V. Vovchenko, L. Bovard, E. R. Most, L. J. Papenfort, S. Schramm, and H. Stoecker, Particles 2, 44 (2019).

[20] Y. Aoki, G. Endrodi, Z. Fodor, S. D. Katz, and K. K. Szabo, Nature (London) 443, 675 (2006).

[21] S. Borsanyi, Z. Fodor, C. Hoelbling, S. D. Katz, S. Krieg, and K. K. Szabo, Phys. Lett. B 730, 99 (2014).

[22] A. Bazavov et al. (HotQCD Collaboration), Phys. Rev. D 90, 094503 (2014).

[23] J. Pochodzalla et al., Phys. Rev. Lett. 75, 1040 (1995).

[24] S. Borsanyi, Z. Fodor, C. Hoelbling, S. D. Katz, S. Krieg, C. Ratti, and K. K. Szabo (Wuppertal-Budapest Collaboration), J. High Energy Phys. 09 (2010) 073.

[25] C. DeTar and T. Kunihiro, Phys. Rev. D 39, 2805 (1989).

[26] T. Hatsuda and M. Prakash, Phys. Lett. B 224, 11 (1989).

[27] P. Papazoglou, J. Schaffner, S. Schramm, D. Zschiesche, H. Stöcker, and W. Greiner, Phys. Rev. C 55, 1499 (1997).

[28] P. Papazoglou, S. Schramm, J. Schaffner-Bielich, H. Stöcker, and W. Greiner, Phys. Rev. C 57, 2576 (1998).

[29] P. Papazoglou, D. Zschiesche, S. Schramm, J. SchaffnerBielich, H. Stöcker, and W. Greiner, Phys. Rev. C 59, 411 (1999).

[30] C. Sasaki and I. Mishustin, Phys. Rev. C 82, 035204 (2010).

[31] J. Steinheimer, S. Schramm, and H. Stoecker, J. Phys. G 38, 035001 (2011).

[32] J. Steinheimer, S. Schramm, and H. Stöcker, Phys. Rev. C 84, 045208 (2011).
[33] V. Dexheimer, J. Steinheimer, R. Negreiros, and S. Schramm, Phys. Rev. C 87, 015804 (2013).

[34] A. Mukherjee, J. Steinheimer, and S. Schramm, Phys. Rev. C 96, 025205 (2017).

[35] A. Motornenko, V. Vovchenko, J. Steinheimer, S. Schramm, and H. Stoecker, Nucl. Phys. A 982, 891 (2019).

[36] G. Aarts, C. Allton, D. De Boni, S. Hands, B. Jager, C. Praki, and J.-I. Skullerud, J. High Energy Phys. 06 (2017) 034.

[37] G. Aarts, C. Allton, D. De Boni, and B. Jäger, Phys. Rev. D 99, 074503 (2019).

[38] V. Dexheimer, S. Schramm, and D. Zschiesche, Phys. Rev. C 77, 025803 (2008).

[39] J. Steinheimer and S. Schramm, Phys. Lett. B 736, 241 (2014).

[40] M. Tanabashi et al. (Particle Data Group), Phys. Rev. D 98, 030001 (2018).

[41] K. Fukushima, Phys. Lett. B 591, 277 (2004).

[42] A. Dumitru, R. D. Pisarski, and D. Zschiesche, Phys. Rev. D 72, 065008 (2005).

[43] K. Fukushima and Y. Hidaka, Phys. Rev. D 75, 036002 (2007).

[44] S. Roessner, T. Hell, C. Ratti, and W. Weise, Nucl. Phys. A 814, 118 (2008).

[45] J. Steinheimer and S. Schramm, Phys. Lett. B 696, 257 (2011).

[46] M. G. Alford, A. Schmitt, K. Rajagopal, and T. Schäfer, Rev. Mod. Phys. 80, 1455 (2008).

[47] K. Rajagopal and F. Wilczek, in At the Frontier of Particle Physics: Handbook of QCD, Vols. 1-3, edited by M. Shifman and B. Ioffe (World Scientific, Singapore, 2000), pp. 2061-2151.

[48] D. Nickel, Phys. Rev. D 80, 074025 (2009).

[49] S. Rößner, C. Ratti, and W. Weise, Phys. Rev. D 75, 034007 (2007).

[50] R. D. Pisarski, Phys. Rev. D 62, 111501(R) (2000).

[51] C. Ratti, M. A. Thaler, and W. Weise, Phys. Rev. D 73, 014019 (2006).

[52] P. G. Reinhard, Rep. Prog. Phys. 52, 439 (1989).

[53] V. Skokov, B. Friman, E. Nakano, K. Redlich, and B. J. Schaefer, Phys. Rev. D 82, 034029 (2010).

[54] K. Fukushima, Phys. Rev. D 77, 114028 (2008); 78, 039902(E) (2008).

[55] M. Albright, J. Kapusta, and C. Young, Phys. Rev. C 90, 024915 (2014).

[56] S. Borsanyi, Z. Fodor, S. D. Katz, S. Krieg, C. Ratti, and K. Szabo, J. High Energy Phys. 01 (2012) 138.

[57] R. Bellwied, S. Borsanyi, Z. Fodor, S. D. Katz, A. Pasztor, C. Ratti, and K. K. Szabo, Phys. Rev. D 92, 114505 (2015).

[58] A. Bazavov et al., Phys. Rev. D 95, 054504 (2017).

[59] A. Bazavov et al. (HotQCD Collaboration), Phys. Rev. D 96, 074510 (2017).

[60] M. A. Stephanov, Phys. Rev. Lett. 102, 032301 (2009).

[61] D. Zschiesche, G. Zeeb, S. Schramm, and H. Stoecker, J. Phys. G 31, 935 (2005).

[62] S. Floerchinger and M. Martinez, Phys. Rev. C 92, 064906 (2015).

[63] A. H. Taub, Phys. Rev. 74, 328 (1948).

[64] K. S. Thorne, Astrophys. J. 179, 897 (1973).

[65] H. Stoecker, W. Greiner, and W. Scheid, Z. Phys. A 286, 121 (1978).

[66] H. Stoecker and W. Greiner, Phys. Rep. 137, 277 (1986).

[67] L. P. Csernai, J. I. Kapusta, and L. D. McLerran, Phys. Rev. Lett. 97, 152303 (2006). 
[68] P. Romatschke and U. Romatschke, Phys. Rev. Lett. 99, 172301 (2007).

[69] J. Steinheimer, M. Bleicher, H. Petersen, S. Schramm, H. Stöcker, and D. Zschiesche, Phys. Rev. C 77, 034901 (2008).

[70] H. G. Baumgardt, J. U. Schott, Y. Sakamoto, E. Schopper, H. Stoecker, J. Hofmann, W. Scheid, and W. Greiner, Z. Phys. A 273, 359 (1975).

[71] H. Stoecker, G. Graebner, J. A. Maruhn, and W. Greiner, Phys. Lett. B 95, 192 (1980).

[72] H. Stoecker, A. A. Ogloblin, and W. Greiner, Z. Phys. A 303, 259 (1981).

[73] H. Stoecker, M. Gyulassy, and J. Boguta, Phys. Lett. B 103, 269 (1981).

[74] D. Hahn and H. Stoecker, Nucl. Phys. A 476, 718 (1988).

[75] A. V. Merdeev, L. M. Satarov, and I. N. Mishustin, Phys. Rev. C 84, 014907 (2011).

[76] K. Fukushima, Phys. Rev. C 91, 044910 (2015).

[77] V. Vovchenko, M. I. Gorenstein, and H. Stoecker, Phys. Rev. Lett. 118, 182301 (2017).

[78] V. Vovchenko, L. Jiang, M. I. Gorenstein, and H. Stoecker, Phys. Rev. C 98, 024910 (2018).

[79] T. Dietrich, S. Bernuzzi, M. Ujevic, and B. Brugmann, Phys. Rev. D 91, 124041 (2015).

[80] D. Radice, S. Bernuzzi, W. Del Pozzo, L. F. Roberts, and C. D. Ott, Astrophys. J. 842, L10 (2017).

[81] C. Greiner, P. Koch, and H. Stöcker, Phys. Rev. Lett. 58, 1825 (1987).
[82] M. Hempel, V. Dexheimer, S. Schramm, and I. Iosilevskiy, Phys. Rev. C 88, 014906 (2013).

[83] R. V. Poberezhnyuk, V. Vovchenko, M. I. Gorenstein, and H. Stoecker, Phys. Rev. C 99, 024907 (2019).

[84] R. C. Tolman, Phys. Rev. 55, 364 (1939).

[85] J. R. Oppenheimer and G. M. Volkoff, Phys. Rev. 55, 374 (1939).

[86] G. Baym, C. Pethick, and P. Sutherland, Astrophys. J. 170, 299 (1971).

[87] S. Benic, D. Blaschke, D. E. Alvarez-Castillo, T. Fischer, and S. Typel, Astron. Astrophys. 577, A40 (2015).

[88] M. A. R. Kaltenborn, N.-U. F. Bastian, and D. B. Blaschke, Phys. Rev. D 96, 056024 (2017).

[89] T. Fischer, N.-U. F. Bastian, M.-R. Wu, P. Baklanov, E. Sorokina, S. Blinnikov, S. Typel, T. Klahn, and D. B. Blaschke, Nat. Astron. 2, 980 (2018).

[90] Y. Song, G. Baym, T. Hatsuda, and T. Kojo, Phys. Rev. D 100, 034018 (2019).

[91] S. Benic, I. Mishustin, and C. Sasaki, Phys. Rev. D 91, 125034 (2015).

[92] M. Marczenko, D. Blaschke, K. Redlich, and C. Sasaki, Phys. Rev. D 98, 103021 (2018).

[93] T. Hinderer, B. D. Lackey, R. N. Lang, and J. S. Read, Phys. Rev. D 81, 123016 (2010).

[94] B. P. Abbott et al. (LIGO Scientific Collaboration and Virgo Collaboration), Phys. Rev. X 9, 011001 (2019). 